\title{
Lower Extremity Joint Posture and Peak Knee Valgus Moment during Side-step Cutting Performed by Males and Females
}

\author{
Chaiyong Jorrakate, PhD, Candidate ${ }^{1)}$, Roongtiwa Vachalathiti, $\mathrm{PhD}^{1)}$, \\ MANTANA VONGSIRINAVARAT, PhD ${ }^{1)}$, SIRIPORN SASIMONTONKUL, PhD ${ }^{2)}$ \\ ${ }^{1)}$ Faculty of Physical Therapy, Mahidol University: Phuttamonthon 4 Rd, Salaya, Phuttamonthon, \\ Nakhon Pathom 73170, Thailand.TEL: +66 2-441-5450,E-mail: ptrvc@mahidol.ac.th \\ ${ }^{2)}$ Faculty of Sport Sciences, Kasetsart University
}

\begin{abstract}
Purpose] To determine the associations between peak knee valgus moment and lower extremity joint angles at initial contact and at peak knee valgus moment during side-step cutting and to investigate their associations with gender. [Subjects and Methods] Twenty healthy subjects and twenty basketball players participated in this study. Three-dimensional kinematics and kinetics of hip, knee and ankle joints were recorded during a side-step cutting protocol by six video cameras and a forceplate. Associations between peak knee valgus moment and three-dimensional hip, knee and ankle joint angles were analyzed using Pearson's product moment correlation. [Results] Peak knee valgus moment was significantly associated with increased hip flexion, knee flexion, ankle inversion and ankle external rotation at initial contact and at peak knee valgus moment. With regard to gender, significant associations between peak knee valgus moment and hip, knee and ankle joint angles were found only in females. [Conclusion] The results provided data on lower extremity joint posture related to peak knee valgus moment during side-step cutting and indicate that females are more sensitive to increased peak knee valgus moment while performing side-step cutting.

Key words: Peak knee valgus moment, Side-step cutting, Lower extremity posture
\end{abstract}

(This article was submitted Jan. 24, 2011, and was accepted Mar. 8, 2011)

\section{INTRODUCTION}

Peak knee valgus moment is considered to be a risk factor predisposing athlete to anterior cruciate ligament (ACL) injury in non-contact episodes, especially females ${ }^{1-6)}$. ACL injury is a common traumatic sport injury and is a potential cause of loss of sport participation, prolonged disability and heightened risk of osteoarthritic knee ${ }^{1)}$. Despite several published studies having revealed the significant valgus moment at the knee joint during side-step cutting in females ${ }^{2,4,7)}$, only a few studies have investigated the association between peak knee valgus moment and the posture of lower extremity joints ${ }^{2,8)}$. McLean et al. ${ }^{2)}$ found that a greater peak knee valgus moment was associated with larger initial hip flexion and internal rotation and with larger initial knee valgus angle during side-step cutting. Sigward and Powers ${ }^{8)}$ studied the loading characteristics of female athletes who exhibited excessive knee valgus moment during side-step cutting. They found that the lateral ground reaction forces, internal foot progression angle, initial hip abduction and internal rotation angles were significantly associated with peak knee valgus moment and the multiple regression analysis showed that together those variables could explain $49 \%$ of the variance in peak knee valgus moment. The results of these two studies suggest that knee valgus moment affects the angle of knee and the adjacent joints at initial contact.

Joint angle at initial contact has been suggested to be the source of a subsequent peak knee valgus moment ${ }^{2}$. However, the angles of the hip, knee and ankle at peak knee valgus moment may also influence the magnitude of peak knee valgus moment. Therefore, the associations between peak knee valgus moment and angles of the hip, knee and ankle joints at peak knee valgus moment during side-step cutting should be considered. It may be possible to minimize knee valgus moment during side-step cutting if the associations between peak knee valgus moment and lower extremity joint postures at peak knee valgus moment are verified.

Therefore, the first purpose of this study was to explore the associations between peak knee valgus moment and hip, knee and ankle joint angles at initial contact and at peak knee valgus moment during side-step cutting. Based on reports that females produce significantly greater knee valgus moment than males during side-step cutting ${ }^{2,4,7)}$, the second purpose of the current study was to further evaluate whether lower extremity joint posture associated with peak knee valgus moment was related to gender. 
Table 1. Subject characteristics, mean (SD)

\begin{tabular}{lcccc}
\hline & $\begin{array}{c}\text { Healthy male } \\
(\mathrm{n}=10)\end{array}$ & $\begin{array}{c}\text { Healthy female } \\
(\mathrm{n}=10)\end{array}$ & $\begin{array}{c}\text { Male basketball athletes } \\
(\mathrm{n}=10)\end{array}$ & $\begin{array}{c}\text { Female basketball athletes } \\
(\mathrm{n}=10)\end{array}$ \\
\hline Age (years) & $20.1(0.9)$ & $21.8(2.6)$ & $19.7(1.2)$ & $20.8(1.3)$ \\
Weight $(\mathrm{kg})$ & $65.4(7.5)$ & $54.3(3.5)$ & $76.1(9.3)$ & $57.0(4.0)$ \\
Height $(\mathrm{cm})$ & $175.9(3.6)$ & $161.4(3.4)$ & $178.3(6.7)$ & $167.7(4.7)$ \\
BMI $\left(\mathrm{kg} / \mathrm{m}^{2}\right)$ & $21.1(1.7)$ & $20.8(1.4)$ & $23.9(2.4)$ & $20.3(1.1)$ \\
Approach running speed $(\mathrm{m} / \mathrm{s})$ & $4.5(0.4)$ & $3.6(0.2)$ & $4.7(0.1)$ & $4.3(0.3)$ \\
Experience (years) & - & - & $8.1(2.4)$ & $7.8(2.2)$ \\
\hline
\end{tabular}

\section{SUBJECTS AND METHODS}

The subjects were 20 healthy subjects ( 10 males and 10 females) and 20 basketball players (10 males and 10 females). The healthy subjects were university students and their activity levels were identified as active by the Habitual Physical Activity Questionnaire ${ }^{9}$. Basketball players in university teams were recruited from various universities in Bangkok. A summary of subject characteristics is shown in Table 1. All subjects were healthy with no current complaints of lower extremity injury and no history of serious injury or operative treatment of the hip, knee and ankle joints.

Three-dimensional kinematic data were recorded using a Vicon 6-video camera analysis system (Oxford Metrics, Oxford, England) at a sampling frequency of $200 \mathrm{~Hz}$. Ground reaction forces were collected by a single force platform $(1000 \mathrm{~Hz})$ embedded within the floor (AMTI, Newton, MA, USA). Video cameras and the force platform were interfaced to the same personal computer in order to synchronize the kinematic and kinetic data.

The procedure of this study was approved by the Mahidol University Institutional Review Board. All subjects were asked to read a participant information sheet and then signed an informed consent form. All testing procedures were performed at the Movement Analysis Laboratory at the Faculty of Physical Therapy, Mahidol University.

Before data collection, weight, height, leg lengths, knee widths and ankle joint widths were measured. Reflective spherical markers (14 $\mathrm{mm}$ in diameter) were placed on anatomical landmarks according to the modified Helen Hayes marker set ${ }^{10)}$ : including front and back of the head, T10 spinous process, acromion processes, lateral humeral condyle, medial and lateral styloid processes, $1 \mathrm{st}$ metacarpophalangeal joints, anterior superior iliac spine, posterior superior iliac spine, lateral femoral condyle and lateral malleoli. Thigh and shank markers were mounted on $5 \mathrm{~cm}$ wands and placed on the thigh and shank with the adhesive tape. Foot markers were directly attached to the shoes. The same marker positions were used for all subjects. All subjects were fitted with the same style of running shoes in order to eliminate the variations caused by different footwear.

Each subject was asked to perform side-step cutting task for 5 trials. The side-step cutting task in the current study was modified from the work of McLean et al. ${ }^{2)}$. Cutting angles were assigned between $35^{\circ}$ and $55^{\circ}$ from the original direction of the approach direction. The cutting range was marked on the floor by tape lines originating from the center of the force platform.

Subjects were instructed to run 5 meters at their fastest speed under the laboratory circumstance and plant their right foot on the force platform, then change the approach direction to the left by putting the left foot within the prescribed range. Subjects were required to continue running following the cutting for approximately 2-3 steps. The side-step cutting trial was considered successful when the subjects planted their right foot on the force platform and placed their left foot in the prescribed range following the cutting. Approach running speed was calculated on the Vicon Workstation by averaging the linear velocity of the T10 marker across 10 frames recorded prior to foot contact on the force platform. Practice trials were allowed until each subject became familiar with the procedures and instrumentation.

Vicon Workstation (Vicon 612) and Plug-in Gait Model software (Oxford Metrics, Oxford, England) were used to quantify three-dimensional hip, knee and ankle joint angles and moments. The joint angles and moments were analyzed during the stance phase (heel strike to toe-off) of side-step cutting. Heel strike and toe-off were defined as the vertical ground reaction force was greater and lower than $10 \mathrm{~N}$, respectively. Kinematic and kinetic data were filtered with a 4th order, zero-lag, low-pass Butterworth filter at $20 \mathrm{~Hz}$ and $30 \mathrm{~Hz}$, respectively. The filter frequencies were selected by performing a residual analysis, fast Fourier analysis and visual inspection of the data. All kinetic data were normalized to body mass and net joint moments were calculated with standard inverse dynamics equations. Joint moments in the current study are referred to the net muscle moment (internal moment).

The variables analysed in this study included peak knee valgus moment and the angles of the hip, knee and ankle joints at initial contact and at peak knee valgus moment in the sagittal, frontal and horizontal planes. To identify the representative data of each subject, 5 trials of hip, knee and ankle joint excursions and three-dimensional ground reaction forces during side-step cutting were plotted and the three most similar trials in joint excursions and ground reaction forces, as determined by visual inspection, were averaged and used as representative data for each subject.

The Kolmogorov Smirnov goodness of fit test was used to test the data distribution. The associations between peak knee valgus moment and hip, knee and ankle joint angles at 
Table 2. Hip, knee and ankle joint angles at initial contact and at peak knee valgus moment in degrees

\begin{tabular}{|c|c|c|c|c|c|c|}
\hline \multirow[b]{2}{*}{ Hip } & \multicolumn{3}{|c|}{ Initial contact } & \multicolumn{3}{|c|}{ At peak } \\
\hline & Sagittal $^{\text {a }}$ & Frontal $^{\mathrm{b}}$ & Horizontal $^{\mathrm{c}}$ & Sagittal $^{\mathrm{a}}$ & Frontal $^{\mathrm{b}}$ & Horizontal $^{\mathrm{c}}$ \\
\hline Healthy males & $57.6(4.9)$ & $-10.7(7.0)$ & $-3.5(8.6)$ & $52.7(5.4)$ & $-9.8(6.7)$ & $-2.5(13.9)$ \\
\hline Healthy females & $47.1(6.8)$ & $-4.0(5.8)$ & $6.4(16.5)$ & $42.0(6.7)$ & $-4.2(5.5)$ & 11.4 (14.6) \\
\hline Male athletes & $59.3(8.1)$ & $-7.6(7.4)$ & $8.3(14.3)$ & $52.6(7.9)$ & $-7.4(7.3)$ & $10.5(14.1)$ \\
\hline \multirow[t]{2}{*}{ Female athletes } & $57.8(10.4)$ & $-2.5(7.0)$ & $11.8(16.0)$ & $51.6(9.7)$ & $-3.4(6.0)$ & $15.4(15.4)$ \\
\hline & \multicolumn{3}{|c|}{ Initial contact } & \multicolumn{3}{|c|}{ At peak } \\
\hline Knee & Sagittal $^{\mathrm{a}}$ & Frontal $^{b}$ & Horizontal $^{\mathrm{c}}$ & Sagittal $^{\mathrm{a}}$ & Frontal $^{\mathrm{b}}$ & Horizontal $^{\mathrm{c}}$ \\
\hline Healthy male & $31.9(5.6)$ & $0.8(6.1)$ & $3.8(13.9)$ & $35.1(6.1)$ & $-0.8(9.0)$ & $1.2(17.6)$ \\
\hline Healthy females & $27.0(9.5)$ & $1.3(7.2)$ & $-3.4(10.2)$ & $28.6(6.8)$ & $2.8(6.8)$ & $-6.9(8.7)$ \\
\hline Male athletes & $32.66(9.0)$ & $3.6(8.6)$ & $3.3(9.8)$ & $34.5(7.3)$ & $4.0(7.6)$ & $1.0(12.1)$ \\
\hline \multirow[t]{2}{*}{ Female athletes } & $34.0(11.7)$ & $3.8(8.6)$ & $3.3(17.7)$ & $35.0(10.2)$ & $5.0(8.1)$ & $0.5(17.4)$ \\
\hline & \multicolumn{3}{|c|}{ Initial contact } & \multicolumn{3}{|c|}{ At peak } \\
\hline Ankle & Sagittal $^{\text {a }}$ & Frontal $^{\mathrm{b}}$ & Horizontal $^{\mathrm{c}}$ & Sagittal $^{\text {a }}$ & Frontal $^{\mathrm{b}}$ & Horizontal $^{\mathrm{c}}$ \\
\hline Healthy males & $4.6(12.3)$ & $1.0(1.6)$ & $-10.2(13.4)$ & $3.1(7.4)$ & $0.8(1.2)$ & $-8.6(12.2)$ \\
\hline Healthy females & $9.1(13.6)$ & $0.8(1.4)$ & $-7.1(9.7)$ & $5.0(10.2)$ & $0.4(1.2)$ & $-5.9(8.0)$ \\
\hline Male athletes & $-1.7(11.6)$ & $1.0(2.1)$ & $-7.4(11.0)$ & $-0.3(7.8)$ & $1.1(1.6)$ & $-8.0(9.0)$ \\
\hline Female athletes & $11.2(8.4)$ & $3.0(2.7)$ & $-17.2(11.5)$ & $7.0(6.7)$ & $2.6(2.7)$ & $-15.0(12.4)$ \\
\hline
\end{tabular}

a: the positive value $(+)=$ hip flexion, knee flexion and dorsiflexion; the negative value $(-)=$ hip extension, knee extension and plantar flexion. b: the positive value $(+)=$ hip adduction, knee varus and ankle inversion; the negative value $(-)=$ hip abduction, knee valgus and ankle eversion. c: the positive value $(+)=$ hip internal rotation, knee internal rotation and ankle internal rotation; the negative value $(-)=$ hip external rotation, knee external rotation and ankle external rotation.

initial contact and at peak knee valgus moment were determined by Pearson's product moment correlation. Statistical analyses were performed using SPSS statistical software (Chicago, IL, USA). Values of $\mathrm{p}<0.05$ were considered significant.

\section{RESULTS}

The angles of hip, knee and ankle joints at initial contact, at peak knee valgus moment and the values of peak knee valgus moment during side-step cutting are shown in Tables 2 and 3.

In the sagittal plane, there were significant associations between peak knee valgus moment and hip and knee flexion at initial contact and at peak knee valgus moment $(\mathrm{p}<0.01)$. In the frontal and horizontal planes, significant associations were found at ankle inversion $(\mathrm{p}<0.05)$ and ankle external rotation $(\mathrm{p}<0.05)$, respectively (Table 4$)$.

The peak knee valgus moment increased as the joint angles increased. Regarding gender, there were no significant associations between peak knee valgus moment and hip, knee and ankle joint angles in male subjects, whereas there were significant associations between peak knee valgus moment and increased hip flexion, increased knee flexion and increased ankle inversion during side-step cutting performed by female subjects (Table 5 ).

\section{DISCUSSION}

The aims of this study were to evaluate the associations between peak knee valgus moment and hip, knee and ankle
Table 3. Peak knee joint moment during side-step cutting, mean (SD)

\begin{tabular}{lc}
\hline Group & Valgus moment $(\mathrm{Nm} / \mathrm{kg})$ \\
\hline Healthy males & $0.8(0.3)$ \\
Healthy females & $0.5(0.3)$ \\
Male athletes & $0.6(0.4)$ \\
Female athletes & $0.6(0.5)$ \\
\hline
\end{tabular}

Table 4. Associations between peak knee valgus moment and angles of the hip, knee and ankle joints during side-step cutting $(n=40)$

\begin{tabular}{llll}
\hline Joint angle & \multicolumn{2}{c}{ Initial contact } & At peak \\
\hline Sagittal & Hip & $\mathrm{r}=0.5^{* *}$ & $\mathrm{r}=0.5^{* *}$ \\
& Knee & $\mathrm{r}=0.6^{* *}$ & $\mathrm{r}=0.6^{* *}$ \\
& Ankle & $\mathrm{r}=0.0$ & $\mathrm{r}=0.2$ \\
\hline Frontal & Hip & $\mathrm{r}=0.1$ & $\mathrm{r}=0.2$ \\
& Knee & $\mathrm{r}=0.1$ & $\mathrm{r}=0.0$ \\
& Ankle & $\mathrm{r}=0.4^{*}$ & $\mathrm{r}=0.4^{*}$ \\
\hline Horizontal & Hip & $\mathrm{r}=0.0$ & $\mathrm{r}=0.1$ \\
& Knee & $\mathrm{r}=0.3$ & $\mathrm{r}=0.2$ \\
& Ankle & $\mathrm{r}=0.3^{*}$ & $\mathrm{r}=0.4^{*}$ \\
\hline
\end{tabular}

$*=\mathrm{p}<0.05, * *=\mathrm{p}<0.01$.

joint angles at initial contact and at peak knee valgus moment during side-step cutting and to investigate whether the lower extremity joint posture associated with peak knee valgus moment was related to gender.

In the sagittal plane, there were significant associations 
Table 5. Correlation coefficient values of peak knee valgus moment and angles of the hip, knee and ankle joints during side-step cutting in males and females

\begin{tabular}{lcccc}
\hline & \multicolumn{4}{c}{ Correlation co-efficient $(\mathrm{r})$} \\
\cline { 2 - 5 } $\begin{array}{l}\text { Joint angle associated } \\
\text { to the peak knee } \\
\text { valgus moment }\end{array}$ & \multicolumn{2}{c}{ Males $(\mathrm{n}=20)$} & \multicolumn{2}{c}{ Females $(\mathrm{n}=20)$} \\
\cline { 2 - 5 } & Initial contact & At peak & Initial contact & At peak \\
\hline Hip flexion & 0.3 & 0.3 & $0.6^{* *}$ & $0.5^{*}$ \\
Knee flexion & 0.3 & 0.4 & $0.7^{* *}$ & $0.7^{* *}$ \\
Ankle inversion & 0.1 & 0.2 & $0.6^{* *}$ & $0.5^{*}$ \\
Ankle external rotation & 0.3 & 0.4 & $0.5^{*}$ & 0.4 \\
\hline
\end{tabular}

$*=\mathrm{p}<0.05, * *=\mathrm{p}<0.01$

between peak knee valgus moment and hip and knee joint angles at initial contact and at peak knee valgus moment during side-step cutting. Associations between approach running speed and hip, knee and ankle joint angles were also investigated. Approach running speed was significantly associated with hip flexion $(\mathrm{r}=0.7, \mathrm{p}<0.05)$ and knee flexion angles at initial contact $(\mathrm{r}=0.5, \mathrm{p}<0.05)$. Flexion of hip and knee joints at initial contact would help subjects to decelerate before changing direction during side-step cutting. According to the hypothesis that small knee joint flexion angle during deceleration causes excessive knee extension load and increase risk of knee extension injury ${ }^{11,12}$, subjects should adopt a hip and knee flexion strategy to avoid excessive knee joint loading, adopting this strategy would decrease the risk of knee extension injury during side-step cutting. However, this strategy might lead to an increased peak knee valgus moment and could lead to ACL injury during side-step cutting.

The major muscles of the knee including the quadriceps and hamstrings muscles are most responsible for active knee stability control in the sagittal plane ${ }^{13)}$. The stability control of the knee in the frontal plane is mainly provided by the passive viscoelasticity system, such as the medial collateral ligament (MCL), the lateral collateral ligament (LCL) and meniscus ${ }^{14)}$. MCL and LCL are slack during knee joint flexion causing knee joint laxity ${ }^{15-17)}$. Laxity of knee joint during knee flexion allows the knee to be moved easily by external loading. Therefore, the peak knee valgus moment is probably produced during knee joint flexion during sidestep and it could be a cause of non-contact ACL injury during side-step cutting.

In the frontal plane, only mild associations between ankle joint inversion at initial contact and at peak knee valgus moment were found in the current study, and it was not clear how ankle joint inversion is associated with peak knee valgus moment during side-step cutting. The displacements of ankle joint inversion during side-step cutting were minimal and no previous study has reported a significant association between peak knee valgus moment and ankle joint angle in the frontal plane. Thus, the associations between peak knee valgus moment and ankle joint inversion during side-step cutting might not be clinically meaningful.

In the horizontal plane, there were significant associations between peak knee valgus moment and ankle external rotation at initial contact and at peak knee valgus moment during side-step cutting. The studies of McLean et al. ${ }^{2)}$ and Sigward and Powers ${ }^{8)}$ demonstrated that peak knee valgus was significantly associated with hip joint internal rotation angle at initial contact. Additionally, Sigward and Powers $^{8)}$ reported an association between peak knee valgus moment and internal foot progression angle. They stated that the hip internal rotation position and internal foot progression angle were adopted because the subjects knew that they had to change their running direction during sidestep cutting. A significant association between peak knee valgus moment and hip internal rotation was not found in the current study even though most of subjects performed hip internal rotation during the initial stance of side-step cutting. Ankle external rotation coupled with hip internal rotation might impart a twisting force to the knee resulting in excessive rotation force to the knee.

The side-step cutting protocol used in the current study was similar to those used in the studies of McLean et al. ${ }^{2)}$ and Sigward and Powers ${ }^{8)}$. McLean et al. ${ }^{2)}$ recruited male and female athletes to participate in their study. Sigward and Powers ${ }^{8)}$ conducted their experiment with female athletes who exhibited excessive valgus moment during side-step cutting. Excessive valgus moment in the study of Sigward and Powers ${ }^{8)}$ was indicated when the peak knee valgus moment during side-step cutting was greater than $0.59 \mathrm{Nm} / \mathrm{kg}$. In the current study, healthy males and females, and male and female athletes were recruited as subjects.

Accordingly, the inconsistencies in results between previous studies and the current study might be explained by the influence of different subject characteristics. The current study purposefully employed a wide range of subject's characteristics in order to identify the lower extremity joint posture related to peak knee valgus moment. Moreover, the current study investigated the associations at 2 events, initial contact and peak knee valgus moment, whereas previous studies $^{2,8)}$ only investigated associations at initial contact during side-step cutting. The similar significant associations between peak knee valgus moment and lower extremity joint posture observed at initial contact and at peak knee valgus moment in the current study strongly suggest that hip joint flexion, knee joint flexion, ankle joint inversion and ankle joint external rotation are related to peak knee valgus moment during side-step cutting. In the comparison with previous results, there were some inconsistent results, 
however, the associations identified in previous studies and the current study were mostly those of hip joint angles. Thus, it could be hypothesized that proximal control of the hip angle may influence peak knee valgus moment during side-step cutting.

Regarding gender, the values of Pearson's correlation coefficients of the significant associations for the female subjects were greater than those for all the subjects $(n=40)$. The significant associations of the female groups might indicate that females are more sensitive to lower extremity joint posture related to peak knee valgus moment or females might adopt lower extremity movement patterns that are different from males to decelerate their body during sidestep cutting. Therefore, the higher correlation coefficients obtained for females suggest a predisposing factor to the risk of non-contact ACL injury during side-step cutting.

The significant associations between hip, knee and ankle joint angles and peak knee valgus moment suggest that hip flexion, knee flexion, ankle inversion and ankle external rotation should be avoided in order to decrease risk of noncontact ACL injury during side-step cutting. A lower extremity joint posture of slight hip flexion, knee flexion and ankle joint in the neutral position at initial contact during side-step cutting would reduce peak knee valgus moment. Additionally, strengthening exercises for hip extensor and knee extensor muscles should be considered for athletes to reduce excessive hip and knee joint flexion, as they would prevent excessive knee valgus moment during side-step cutting.

The significant associations between peak knee valgus moment and lower extremity joint posture observed in females indicate a predisposing factor to a greater incidence of non-contact ACL injury. Accordingly, a neuromuscular training program and side-step cutting strategy should be concerned and properly designed for females.

Data collection during side-step cutting in the current study was conducted in a laboratory. The situation simulated in the laboratory is different from real situations, and subjects may perform side-step cutting tasks differently in an actual sport game. Further research should be conducted on the sport field in order to determine lower extremity mechanics while subjects perform side-step cutting tasks in real situations.

Electromyographic analysis should be used in the future studies in order to determine the neuromuscular control and activation patterns of the muscles around the hip, knee and ankle joints during side-step cutting. Likewise, the effect of a neuromuscular training program on reduction of peak knee valgus moment during side-step cutting would be interesting to study in order to indicate the appropriateness of a training program for athletes.

In conclusion, this study investigated the associations between lower extremity joint posture and peak knee valgus moment in both male and female, healthy and athlete subjects. Peak knee valgus moment was significantly associated with hip flexion, knee flexion, ankle inversion and external rotation at initial contact and at peak knee valgus moment during side-step cutting. When stratified by gender, females demonstrated significant associations between peak knee valgus moment and hip, knee and ankle joint angles at initial contact and at peak knee valgus moment during side-step cutting but no significant associations were found for male subjects.

\section{ACKNOWLEDGEMENT}

This research was funded by the Higher Education Commission, Ministry of Education, Thailand.

\section{REFERENCES}

1) Hewett TE, Ford KR, Myer GD: Anterior cruciate ligament injuries in female athletes: part 2: a meta-analysis of neuromuscular interventions aimed at injury prevention. Am J Sports Med, 2005, 34: 1-9.

2) McLean SG, Huang X, Van den Bogert AJ: Association between lower extremity posture at contact and peak knee valgus moment during sidestepping: implications for ACL injury. Clin Biomech, 2005, 20: 863-870.

3) Pollard CD, Davis IM, Hamill J: Influence of gender on hip and knee mechanics during a randomly cued cutting maneuver. Clin Biomech, 2004, 19: 1022-1031.

4) Sigward SM, Powers CM: The influence of gender on knee kinematics, kinetics and muscle activation patterns during side-step cutting. Clin Biomech, 2006, 21: 41-48.

5) Hewett TE, Myer GD, Ford KR, et al.: Biomechanical measures of neuromuscular control and valgus loading of the knee predict anterior cruciate ligament injury risk in female athletes: a prospective study. Am J Sports Med, 2005, 33: 492-501.

6) Ford KR, Myer GD, Toms HE, et al.: Gender differences in kinematics of unanticipated cutting in young athletes. Med Sci Sports Exerc, 2005, 37: 124 129.

7) McLean SG, Huang X, Su A, et al.: Sagittal plane biomechanics cannot injure the ACL during sidestep cutting. Clin Biomech, 2004, 19: 828-838.

8) Sigward SM, Powers CM: Loading characteristics of females exhibiting excessive valgus moments during cutting. Clin Biomech, 2007, 22: 827-833.

9) Baecke JAH, Burema J, Frijters JER: A short questionnaire for the measurement of habitual physical activity in epidemiological studies. Am J Clin Nutr, 1982, 36: 936-942.

10) Kadaba MP, Ramakrishnan HK, Wootten ME, et al.: Repeatability of kinematic, kinetic and electromyographic data in normal adult gait. J Orthop Res 1989, 7: 849-860.

11) Hewett TE, Myer GD, Ford KR: Anterior cruciate ligament injuries in female athletes: part 1, mechanisms and risk factors. Am J Sports Med, 2006, 34 : 299-311.

12) Davis I, Ireland ML, Hanaki S: ACL injuries - the gender bias (ACL supplement: consensus statement). J Orthop Sports Phys Ther, 2007, 37: A1A7.

13) Solomonow M, D'Ambrosia R: Neural reflex arcs and muscle control of knee stability and motion. In: The knee. St. Louis: Mosby-Year Book, 1994, pp $107-120$.

14) Wasielewski RC: Surgical anatomy of the knee. In: The adult knee. Philadelphia: Lippincott Williams \& Willkins, 2003, pp 55-79.

15) Gardiner JC, Weiss JA, Rosenberg TD: Strain in the human medial collateral ligament during valgus loading of the knee. Clin Orthop Relat Res, 2001, 391: $266-274$.

16) Meister BR, Michael SP, Moyer RA, et al.: Anatomy and kinematics of the lateral collateral ligament of the knee. Am J Sports Med, 2000, 28: 869-878.

17) Sugita T, Amis AA: Anatomic and biomechanical study of the lateral collateral and popliteofibular ligaments. Am J Sports Med, 2001, 29: 466472 . 